\title{
Experimental Demonstration and Analytic Derivation of Chromatic Dispersion Monitoring Technique Based on Clock-frequency Component
}

\author{
Sung-Man Kim* \\ Department of Electronic Engineering, Kyungsung University, Daeyeon-3-dong, Nam-gu, Busan 608-736, Korea
}

(Received July 12, 2012 : revised August 21, 2012 : accepted August 22, 2012)

\begin{abstract}
In an earlier work, we proposed the chromatic dispersion monitoring technique of non-return to zero (NRZ) signal based on clock-frequency component (CFC) through numerical simulations. However, we have not yet shown any experimental demonstration or analytic derivation of it. In this paper, we show an experimental demonstration and analytic derivation of the proposed chromatic dispersion monitoring technique. We confirm that the experimental results and the analytic results correspond with the simulation results. We also demonstrate that monitoring range and accuracy can be improved by using a simple clock-extraction method.
\end{abstract}

Keywords: Chromatic dispersion monitoring, Clock-frequency component, Non-return to zero (NRZ) OCIS codes : (060.2330) Fiber optics communications; (060.2360) Fiber optics links and subsystems

\section{INTRODUCTION}

In the high-speed optical communication systems with $>40$ $\mathrm{Gb} / \mathrm{s}$ per channel, it is essential to monitor and compensate for chromatic dispersion that can be changed by temperature variation [1] or dynamic reconfiguration of the optical network. Until now, there have been substantial efforts to develop the chromatic dispersion monitoring techniques, which include the techniques based on clock-frequency component (CFC), subcarrier tone, and phase-modulation to amplitudemodulation conversion [2-11]. Among the techniques, the technique using the power change of the CFC in the received signal seems to be the simplest because it does not need to add any extra modulation to the data signal [7-11].

However, the non-return-to-zero (NRZ) signal widely used in optical communication systems originally does not have a CFC. Therefore, if we want to use a CFC for chromatic dispersion monitoring in an NRZ signal, we need a clockextraction process. In an earlier work, we proposed a couple of efficient clock-extraction methods of NRZ signal for chromatic dispersion monitoring through numerical simulations [12]. In another earlier work, we showed that CFC of carriersuppressed return-to-zero (CSRZ) signal can be used for chromatic dispersion monitoring through numerical simulations [13]. In the earlier works, we evaluated the dispersion monitoring performance of the CFCs with two criteria: monitoring range and monitoring sensitivity to dispersion.

However, we have not yet shown any experimental demonstration or analytic derivation for the proposed techniques. Therefore, in this paper, we show an experimental demonstration and analytic derivation of the proposed chromatic dispersion monitoring technique based on CFC in NRZ signal. We conduct the experiment to obtain the experimental value of monitoring range and monitoring sensitivity and compare them with the simulation results and the analytic results. We also want to see if monitoring range and accuracy can be improved by using an efficient clock-extraction method.

\section{EXPERIMENTAL SETUP}

Figure 1 shows the experimental setup of chromatic dispersion monitoring based on CFC in the $10-\mathrm{Gb} / \mathrm{s}$ NRZ signal. To accumulate chromatic dispersion, we transmitted an optical NRZ signal up to $200 \mathrm{~km}$ through a single-mode fiber. At the receiver site, the optical signal is converted into an electrical signal through a $10-\mathrm{Gb} / \mathrm{s}$ photodiode (PD), and the electrical signal is amplified by using a $10-\mathrm{Gb} / \mathrm{s}$ electrical amplifier. And then, the amplified electrical signal is sent

\footnotetext{
*Corresponding author: sungman@ks.ac.kr

Color versions of one or more of the figures in this paper are available online.
} 


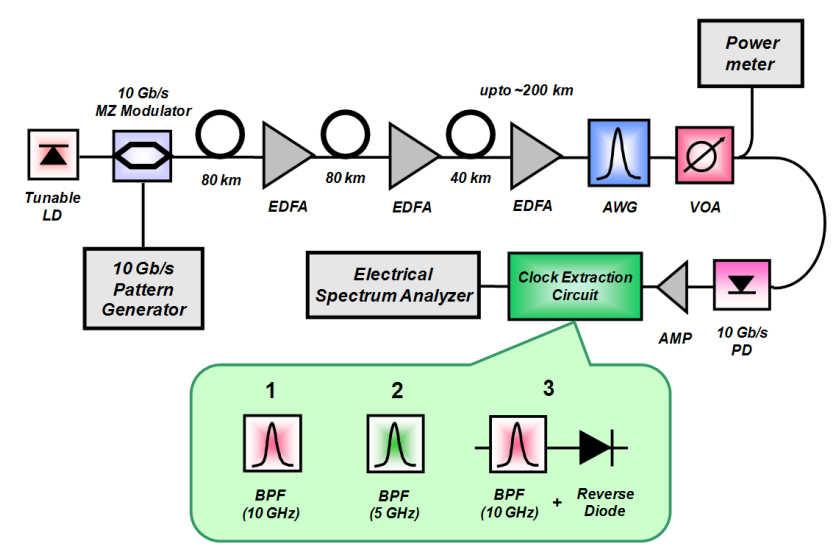

FIG. 1. Experimental setup of chromatic dispersion monitoring using CFCs extracted by three clock-extraction methods.

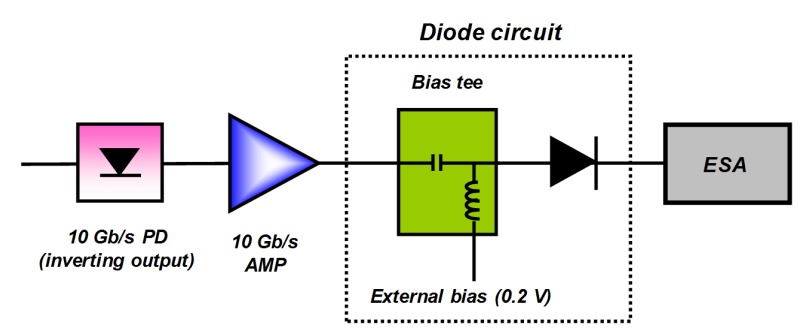

FIG. 2. Implementation of the reverse diode function.

into the clock-extraction circuit where three clock-extraction methods are used.

In the experiment, we implemented three clock-extraction methods: (1) the pure $\mathrm{CFC}$, i.e. a $10-\mathrm{GHz}$ component in this experiment (referred to as " $\mathrm{Cl}$ " in Kim et al. [12]), (2) the half $\mathrm{CFC}$, i.e. a 5-GHz component in this experiment (referred to as "C22" in Kim et al. [12]), and (3) the $\mathrm{CFC}$ after a reverse diode function (referred to as " $\mathrm{C} 5$ " in Kim et al. [12]). The reverse diode function was implemented by using a schottky diode. The schottky diode has a barrier voltage of $0.2 \mathrm{~V}$, thus we added a DC bias of $0.2 \mathrm{~V}$ to the input signal of the reverse diode by using a bias tee. The implementation of the reverse diode function in the experiment is shown in Fig. 2. Because we used an inverting output port of the PD, the direction of the diode is forward in Fig. 2. We measured the CFC power by using an electrical spectrum analyzer (ESA) with a resolution bandwidth (BW) of $5 \mathrm{MHz}$. Note that each clock-extraction circuit is very simple and can be easily implemented.

\section{EXPERIMENTAL AND SIMULATION RESULTS}

Using the experimental setup in Fig. 1, we measured the CFC powers of three clock-extraction methods as a function of chromatic dispersion. The experimental results of the pure CFC, the half CFC, and the CFC after a reverse

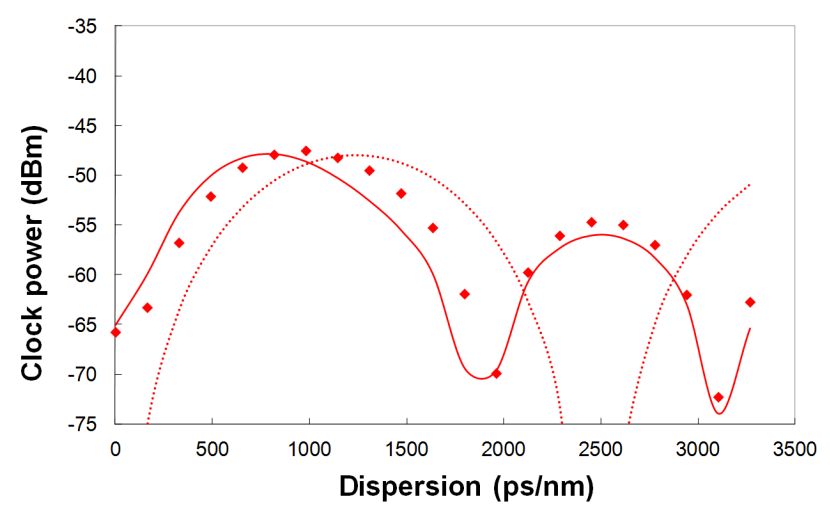

FIG. 3. Result of the pure CFC (solid mark: experimental result, solid line: simulation result, and dotted line: analytic result).

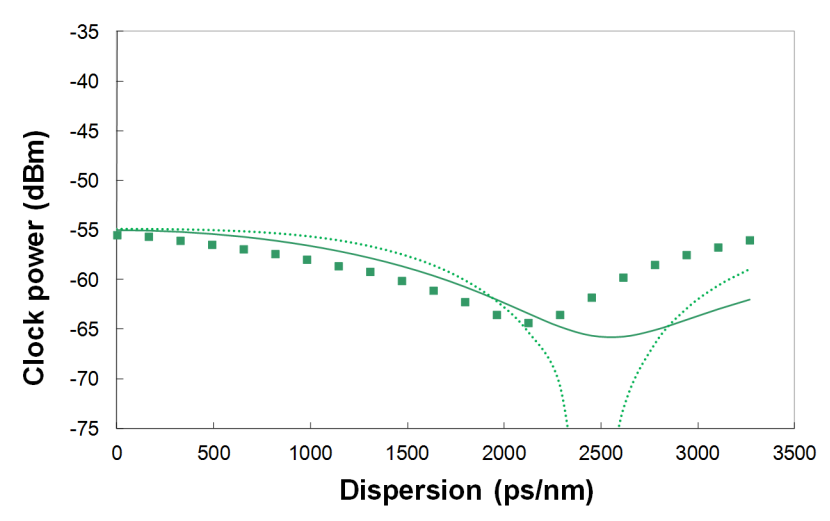

FIG. 4. Result of the half CFC (solid mark: experimental result, solid line: simulation result, and dotted line: analytic result).

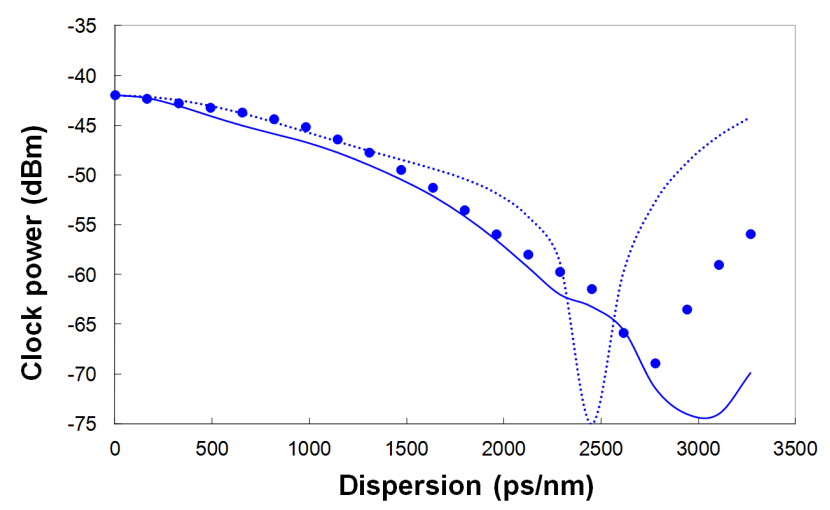

FIG. 5. Result of the CFC after a reverse diode function (solid mark: experimental result, solid line: simulation result, and dotted line: analytic result).

diode function are shown in Fig. 3, Fig. 4, and Fig. 5, respectively. The solid lines in the figures are the simulation results obtained by modeling the electrical BWs of the PD and the electrical amplifier as a one-pole low-pass filter (LPF). The dotted line in the figures are the analytic results, which will be discussed later. We can recognize that the 
experimental results correspond with the simulation results quite well.

In the experimental results, the monitoring ranges of the pure CFC, the half CFC, and the CFC after a reverse diode function are $1000 \mathrm{ps} / \mathrm{nm}, 2100 \mathrm{ps} / \mathrm{nm}$, and $2800 \mathrm{ps} / \mathrm{nm}$, respectively. Their simulation results are $820 \mathrm{ps} / \mathrm{nm}, 2600$ $\mathrm{ps} / \mathrm{nm}$, and $3100 \mathrm{ps} / \mathrm{nm}$, respectively. Even though the experimental results of monitoring range are not exactly same as the simulation results, the tendency of each clock-extraction method corresponds with the simulation results.

In the experiment, we also measured the monitoring accuracy of each CFC. We measured the noise power and CFC power at each point, thus we can define the monitoring accuracy as the amount of chromatic dispersion which corresponds to the noise variation of the CFC power. In the experiment, the resolution $\mathrm{BW}$, the video $\mathrm{BW}$, and sweep time of the ESA were $5 \mathrm{MHz}, 1 \mathrm{kHz}$, and $1 \mathrm{~s}$, respectively. In the experimental results, the monitoring accuracies of the pure CFC, the half CFC, and the CFC after a reverse diode function are $<12 \mathrm{ps} / \mathrm{nm},<200$ $\mathrm{ps} / \mathrm{nm}$, and $<7 \mathrm{ps} / \mathrm{nm}$, respectively. The simulation results are $1.5 \mathrm{~A}, 160 \mathrm{~A}$, and $1.0 \mathrm{~A}$ (A is an arbitrary constant), respectively, when we assume that the noise power of each clock-extraction method is identical. We can recognize that the experimental results of monitoring accuracies correspond with the simulation results quite well. The experimental results and simulation results are summarized in Table 1 and Table 2, respectively.

Among the three CFCs that we conducted the experiment, the CFC after a reverse diode function gives the widest monitoring range and the best monitoring accuracy. This result also shows that the monitoring range and accuracy can be improved by using a suitable clock-extraction

TABLE 1. Summary of experimental results

\begin{tabular}{ccc}
\hline \hline $\begin{array}{c}\text { Clock-extraction } \\
\text { methods }\end{array}$ & $\begin{array}{c}\text { Monitoring } \\
\text { Range }\end{array}$ & $\begin{array}{c}\text { Monitoring } \\
\text { Accuracy }\end{array}$ \\
\hline $\begin{array}{c}\text { Pure CFC } \\
\text { ("C1" in [12]) }\end{array}$ & $\pm 1000 \mathrm{ps} / \mathrm{nm}$ & $<12 \mathrm{ps} / \mathrm{nm}$ \\
\hline $\begin{array}{c}\text { Half CFC } \\
\text { ("C2" in [12]) }\end{array}$ & $\pm 2100 \mathrm{ps} / \mathrm{nm}$ & $<200 \mathrm{ps} / \mathrm{nm}$ \\
\hline $\begin{array}{c}\text { Reverse diode } \\
\text { ("C5" in [12]) }\end{array}$ & $\pm 2800 \mathrm{ps} / \mathrm{nm}$ & $<7 \mathrm{ps} / \mathrm{nm}$ \\
\hline
\end{tabular}

TABLE 2. Summary of simulation results

\begin{tabular}{ccc}
\hline $\begin{array}{c}\text { Clock-extraction } \\
\text { methods }\end{array}$ & $\begin{array}{c}\text { Monitoring } \\
\text { Range }\end{array}$ & $\begin{array}{c}\text { Monitoring } \\
\text { Accuracy }\end{array}$ \\
\hline $\begin{array}{c}\text { Pure CFC } \\
\text { ("C1" in [12]) }\end{array}$ & $\pm 820 \mathrm{ps} / \mathrm{nm}$ & $1.5 \mathrm{~A}$ \\
\hline $\begin{array}{c}\text { Half CFC } \\
\text { ("C2" in [12]) }\end{array}$ & $\pm 2600 \mathrm{ps} / \mathrm{nm}$ & $160 \mathrm{~A}$ \\
\hline $\begin{array}{c}\text { Reverse diode } \\
\text { ("C5" in [12]) }\end{array}$ & $\pm 3100 \mathrm{ps} / \mathrm{nm}$ & $\begin{array}{c}1.0 \mathrm{~A} \\
\text { (A is an arbitrary } \\
\text { constant) }\end{array}$ \\
\hline
\end{tabular}

method. And the clock-extraction circuit for the reverse diode function is so simple; a schottky diode (in reverse direction) and a band-pass filter.

\section{ANALYTIC DERIVATION OF CFC}

In this section, we will derive the analytic expression of the pure CFC, the half CFC, and the CFC after a reverse diode function. Fig. 6 shows our analytic model of optical transmission system for chromatic dispersion monitoring. At the transmitter, we consider a MZ modulator as a typical optical transmitter in our analytic derivation. And, for a simple mathematical expression, we assume that the input NRZ signal can be approximated by a sine wave because a bandwidth-limited NRZ signal is similar to a sine wave when the bit pattern is alternating zeros and ones and a sine wave input is sufficient for the analytic derivation of $\mathrm{CFC}$ as a function of chromatic dispersion [14]. In the model of optical fiber, we consider the chromatic dispersion but ignore the fiber loss because it is not important from the viewpoint of chromatic dispersion monitoring and it will be compensated by an optical amplifier.

At the receiver, we mathematically consider the functions of a photodiode, an AC coupling, a low-pass filter (LPF) as a function of a typical model of an electrical receiver circuit, a clock-extraction process, and a band-pass filter (BPF) for measuring the CFC. In the earlier work, we categorized eleven clock-extraction methods of NRZ signal according to the clock-extraction process, as shown in Fig. 7 [12]. However, we will only show the analytic derivation of $\mathrm{C} 1$ (the pure $\mathrm{CFC}$ ), $\mathrm{C} 2$ (the half $\mathrm{CFC}$ ), and $\mathrm{C} 5$ (the CFC after a reverse diode function) among the eleven clock-extraction methods in Fig. 7.

As the first step of the analytic expression, we consider the MZ modulator. The characteristic of a MZ modulator with the chirpless modulation and at the "normal" bias $\mathrm{V}_{\mathrm{dc}}$ $=\mathrm{V}_{\pi} / 2$, where $\mathrm{V}_{\pi}$ is the modulator extinction voltage, the transfer function is given by [15]

$$
F(x)=\sin \left(x+\frac{\pi}{4}\right)=\frac{\cos (x)+\sin (x)}{\sqrt{2}} .
$$

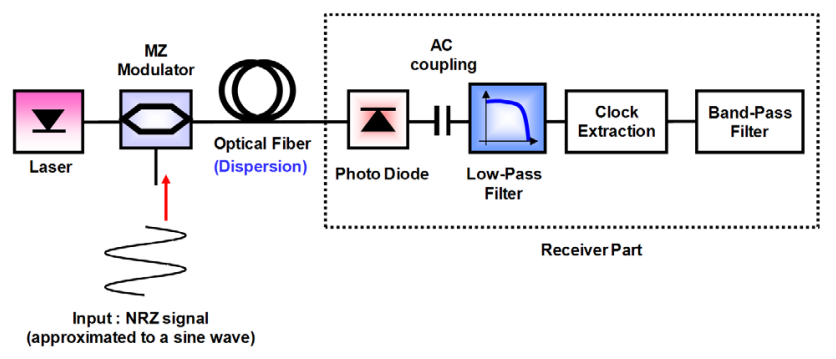

FIG. 6. Analytical model of optical transmission system for CFC. 


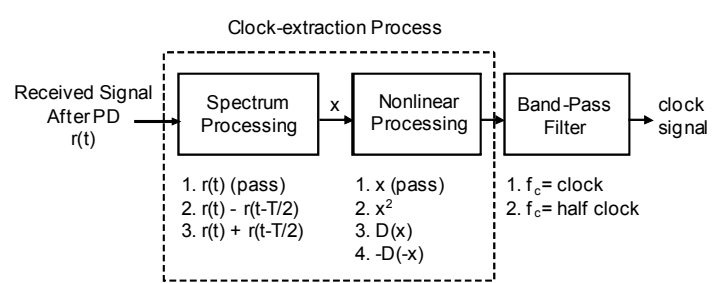

\begin{tabular}{|c|c|c|c|}
\hline $\begin{array}{c}\text { Spectrum } \\
\text { Processing }\end{array}$ & $\begin{array}{c}\text { Nonlinear } \\
\text { Processing }\end{array}$ & $\begin{array}{l}\text { Center freq. } \\
\text { of BPF }\end{array}$ & Mark \\
\hline \multirow{2}{*}{$\begin{array}{c}\mathrm{r}(\mathrm{t}) \\
\text { (pass) }\end{array}$} & \multirow{2}{*}{$\begin{array}{c}\mathrm{X} \\
\text { (pass) } \\
\end{array}$} & clock & C1 \\
\hline & & half clock & $\mathrm{C} 2$ \\
\hline \multirow{3}{*}{$\begin{array}{l}\mathrm{r}(\mathrm{t}) \\
\text { (pass) }\end{array}$} & $x^{2}$ & clock & $\mathrm{C} 3$ \\
\hline & $\mathrm{D}(\mathrm{x})$ & clock & $\mathrm{C} 4$ \\
\hline & $-D(-x)$ & clock & C5 \\
\hline \multirow{3}{*}{$r(t)-r(t-T / 2)$} & $x^{2}$ & clock & $\mathrm{C} 6$ \\
\hline & $\mathrm{D}(\mathrm{x})$ & clock & $\mathrm{C} 7$ \\
\hline & $-D(-x)$ & clock & $\mathrm{C} 8$ \\
\hline \multirow{3}{*}{$r(t)+r(t-T / 2)$} & $x^{2}$ & clock & $\mathrm{C9}$ \\
\hline & $D(x)$ & clock & C10 \\
\hline & $-D(-x)$ & clock & C11 \\
\hline
\end{tabular}

FIG. 7. Clock-extraction methods of NRZ signal [12].

As we mentioned before, we assume that the input NRZ signal can be approximated by a sine wave with a half clock frequency [14]. Thus the input NRZ signal, $i(t)$, of the MZ modulator can be written as

$$
i(t)=\frac{\pi}{4} \cos \left(\frac{\omega_{0} t}{2}\right)
$$

where $\omega_{0} / 2 \pi$ is the clock frequency. When the input signal of the MZ modulator is given by Eq. (2), the output optical electric field of the MZ modulator then becomes:

$$
\begin{aligned}
a(0, t) & =F(i(t))=\frac{\cos (i(t))+\sin (i(t))}{\sqrt{2}} \\
& =\frac{\cos \left(\frac{\pi}{4} \cos \left(\frac{\omega_{0} t}{2}\right)\right)+\sin \left(\frac{\pi}{4} \cos \left(\frac{\omega_{0} t}{2}\right)\right)}{\sqrt{2}} . \\
& \approx \frac{\sqrt{2}+1}{4}+\frac{1}{2} \cos \left(\frac{\omega_{0} t}{2}\right)-\frac{\sqrt{2}-1}{4} \cos \left(\omega_{0} t\right)
\end{aligned}
$$

In the last line of Eq. (3), the first term is a DC component, the second term is the half CFC, and the third term is the CFC in optical electric field. Then, we need to consider the effect of chromatic dispersion. The optical electric field after the transmission through an optical fiber with a length of $\mathrm{z}$ and a chromatic dispersion of $\mathrm{D}=$ $2 \pi \mathrm{c} \cdot \beta_{2} / \lambda^{2}$, ignoring the fiber loss, becomes [16]:

$$
\begin{aligned}
a(z, t) & =\frac{\sqrt{2}+1}{4}+\frac{1}{2} \cos \left(\frac{\omega_{0} t}{2}\right) \cdot \exp \left(\frac{i}{2}\left(\frac{\omega_{0}}{2}\right)^{2} \beta_{2} z\right)-\frac{\sqrt{2}-1}{4} \cos \left(\omega_{0} t\right) \cdot \exp \left(\frac{i}{2} \omega_{0}^{2} \beta_{2} z\right) \\
& =\frac{\sqrt{2}+1}{4}+\frac{1}{2} \cos \left(\frac{\omega_{0} t}{2}\right) \cdot \exp \left(\frac{i}{8} \omega_{0}^{2} \beta_{2} z\right)-\frac{\sqrt{2}-1}{4} \cos \left(\omega_{0} t\right) \cdot \exp \left(\frac{i}{2} \omega_{0}^{2} \beta_{2} z\right) .
\end{aligned}
$$

Due to the square law detection of the $\mathrm{PD}$, the received electrical signal after the PD becomes:

$$
\begin{aligned}
&|a(z, t)|^{2}=a(z, t) \times a(z, t)^{*} \\
&=\frac{13+2 \sqrt{2}}{32} \\
&+\left\{\frac{\sqrt{2}+1}{4} \times \cos \left(\frac{\omega_{0}^{2} \beta_{2} z}{8}\right)-\frac{\sqrt{2}-1}{8} \times \cos \left(\frac{3 \omega_{0}^{2} \beta_{2} z}{8}\right)\right\} \cdot \cos \left(\frac{\omega_{0} t}{2}\right) \\
&+\frac{1}{8} \cdot\left\{1-\cos \left(\frac{\omega_{0}^{2} \beta_{2} z}{2}\right)\right\} \cdot \cos \left(\omega_{0} t\right) \\
&-\frac{\sqrt{2}-1}{8} \cdot \cos \left(\frac{3 \omega_{0}^{2} \beta_{2} z}{8}\right) \cdot \cos \left(\frac{3 \omega_{0} t}{2}\right) \\
&+\frac{3-2 \sqrt{2}}{32} \cdot \cos \left(2 \omega_{0} t\right)
\end{aligned}
$$

In Eq. (5), the first term is a DC component, the second term is the half CFC, the third term is the CFC, the fourth term is the $3 / 2$ CFC, and the last term is the double CFC. Here, we can obtain the analytic expression of the clockextraction methods, $\mathrm{C} 1$ and $\mathrm{C} 2 . \mathrm{C} 1$ is the $\mathrm{CFC}$ of the received signal, i.e., the third term of Eq. (5), and $\mathrm{C} 2$ is the half CFC of the received signal, i.e., the second term of the Eq. (5):

$$
\begin{aligned}
C 1 & \equiv \frac{1}{8} \times\left\{1-\cos \left(\frac{\omega_{0}^{2} \beta_{2} z}{2}\right)\right\}=\frac{1}{8} \times\left\{1-\cos \left(2 \pi^{2} \frac{z}{L_{D}}\right)\right\}, \\
C 2 & \equiv \frac{\sqrt{2}+1}{4} \times \cos \left(\frac{\omega_{0}^{2} \beta_{2} z}{8}\right)-\frac{\sqrt{2}-1}{8} \times \cos \left(\frac{3 \omega_{0}^{2} \beta_{2} z}{8}\right) \\
& =\frac{\sqrt{2}+1}{4} \times \cos \left(\frac{\pi^{2}}{2} \frac{z}{L_{D}}\right)-\frac{\sqrt{2}-1}{8} \times \cos \left(\frac{3 \pi^{2}}{2} \frac{z}{L_{D}}\right),
\end{aligned}
$$

where $L_{D}$ is the dispersion length and is given by [14]

$$
L_{D}=\frac{T_{0}^{2}}{\beta_{2}}=\frac{4 \pi^{2}}{\omega_{0}^{2} \beta_{2}} .
$$

Now we go back to Eq. (5) to derive the analytic expressions of C5. In the nonlinear processing of Fig. 2, the amplitude of each spectrum component is important. However, in the real situation or simulation, the most NRZ optical receivers have a limited bandwidth of [0.75 $\times$ bit rate] typically, so we need to consider the LPF-like characteristic of the receiver. So we may ignore the high frequency components higher than the CFC in Eq. (5). We also need to consider the $\mathrm{AC}$ coupling of the most optical receivers; we have to remove the $\mathrm{DC}$ component. Therefore, we remove the DC component, the $3 / 2 \mathrm{CFC}$, and the double CFC in Eq. (5) and insert a constant to consider the LPF transfer function of the optical receiver. Then the received AC-coupled signal becomes: 


$$
\begin{aligned}
& r(t)=\left(|a(z, t)|^{2}\right)_{A C} \\
& =\alpha_{1 / 2} \cdot\left\{\frac{\sqrt{2}+1}{4} \times \cos \left(\frac{\omega_{0}^{2} \beta_{2} z}{8}\right)-\frac{\sqrt{2}-1}{8} \times \cos \left(\frac{3 \omega_{0}^{2} \beta_{2} z}{8}\right)\right\} \cdot \cos \left(\frac{\omega_{0} t}{2}\right) \\
& +\alpha_{1} \cdot \frac{1}{8} \times\left\{1-\cos \left(\frac{\omega_{0}^{2} \beta_{2} z}{2}\right)\right\} \cdot \cos \left(\omega_{0} t\right) \\
& =A \cdot \cos \left(\frac{\omega_{0} t}{2}\right)+B \cdot \cos \left(\omega_{0} t\right) \text {, } \\
& \text { where } C 2 \equiv A=\alpha_{1 / 2} \cdot\left\{\frac{\sqrt{2}+1}{4} \times \cos \left(\frac{\omega_{0}^{2} \beta_{2} z}{8}\right)-\frac{\sqrt{2}-1}{8} \times \cos \left(\frac{3 \omega_{0}^{2} \beta_{z} z}{8}\right)\right\} \text {, } \\
& C 1 \equiv B=\alpha_{1} \cdot \frac{1}{8} \times\left\{1-\cos \left(\frac{\omega_{0}^{2} \beta_{2} z}{2}\right)\right\}
\end{aligned}
$$

In Eq. (9), $\alpha_{1 / 2}$ and $\alpha_{1}$ are the coefficients to consider the amplitude transfer function of the LPF, and $\mathrm{A}$ and $\mathrm{B}$ are the $1 / 2 \mathrm{CFC}$ and the $\mathrm{CFC}$, i.e. $\mathrm{C} 2$ and $\mathrm{C} 1$, respectively. Here, the Butterworth filter is considered as the LPF characteristic of the receiver. The transfer function of the Butterworth filter as a function of angular frequency, $\omega$, is given by

$$
|G(j \omega)|=\frac{1}{\sqrt{1+\omega^{2 n}}}
$$

where $\mathrm{n}$ is the order of the filter. Thus $\alpha_{1 / 2}$ is 0.96 and $\alpha_{1}$ is 0.39 for the third order Butterworth filter.

Now we will derive the analytic expression of $\mathrm{C} 5$, the CFC after a reverse diode function. C5 has a reverse diode function, which is quite difficult to get an analytic expression for. For better understanding, we illustrated the received signal, $r(t)$, when the signal is transmitted by $z=0.1 L_{D}$ in Fig. 8(a). If this signal is passed through a reverse diode function, the result will be the waveform in Fig. 8(b). Then we can get an analytic expression of CFC of the waveform in Fig. 8(b) using Fourier's theorem [17].

Because we defined the input NRZ signal, $i(t)$, as a cosine wave, the received signal, $r(t)$, is an even function.
If we want to get an Fourier series of Fig. 8(b), we need to get the zero crossing time, $\mathrm{t}_{0}$, when $\mathrm{r}\left(\mathrm{t}_{0}\right)=0$. Therefore, we will solve the question below:

$$
r\left(t_{0}\right)=A \cdot \cos \left(\frac{\omega_{0} t_{0}}{2}\right)+B \cdot \cos \left(\omega_{0} t_{0}\right)=0
$$

Then zero crossing time, $t_{0}$, can be solved from Eq. (11) and is given by

$$
t_{0}= \begin{cases}\frac{2}{\omega_{0}} \operatorname{Arccos}\left(\frac{-A+\sqrt{A^{2}+8 B^{2}}}{4 B}\right), & z / L_{D} \leq 0.31 \\ \frac{2}{\omega_{0}} \operatorname{Arc} \cos \left(\frac{-A-\sqrt{A^{2}+8 B^{2}}}{4 B}\right), & z / L_{D}>0.31\end{cases}
$$

Now we can get the expression of C5, the CFC after a reverse diode function by using Fourier's theorem [17].

$$
\begin{aligned}
C 5 & =\frac{4}{T} \int_{t_{0}}^{T / 2} r(t) \cdot \cos \left(\omega_{0} t\right) \cdot d t \\
& =\frac{\omega_{0}}{\pi} \int_{t_{0}}^{2 \pi / \omega_{0}}\left\{A \cdot \cos \left(\frac{\omega_{0} t}{2}\right)+B \cdot \cos \left(\omega_{0} t\right)\right\} \cdot \cos \left(\omega_{0} t\right) \cdot d t \\
& =\frac{-1}{2 \pi}\left\{A \cdot\left(2 \sin \left(\frac{\omega_{0} t_{0}}{2}\right)+\frac{2}{3} \sin \left(\frac{3 \omega_{0} t_{0}}{2}\right)\right)+B \cdot\left(\omega_{0} t_{0}-2 \pi+\frac{1}{2} \sin \left(2 \omega_{0} t_{0}\right)\right)\right\}
\end{aligned}
$$

In conclusion, the analytic expressions of the pure CFC, the half CFC, and the CFC after a reverse diode function are derived in Eq. (6), (7), and (13), respectively. The analytic results are shown as a dotted line in Fig. 3, 4, and 5. We approximated the input NRZ signal to a simple cosine wave and assumed the repeated " 0101 " bit pattern in analytic derivation while we considered the real shape of the NRZ signal and a $2^{7}-1$ PRBS (pseudo random binary sequence) pattern in the numerical simulations and the experiments. This causes a mismatch between the analytic results and other results. The similar mismatch of

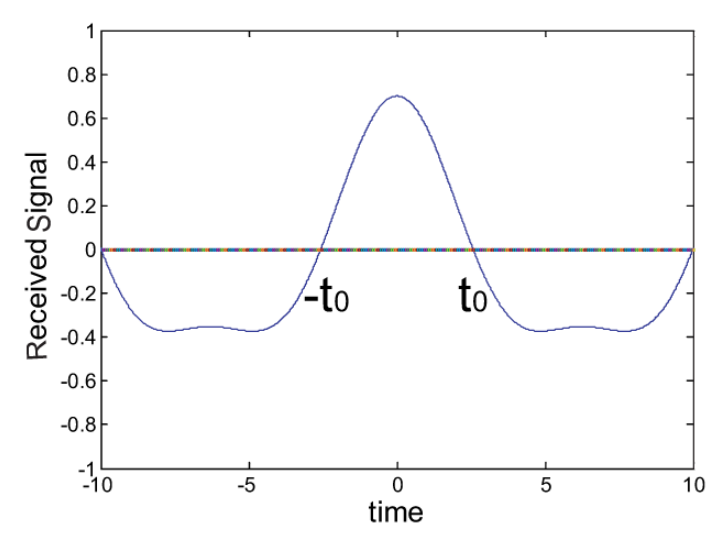

(a)

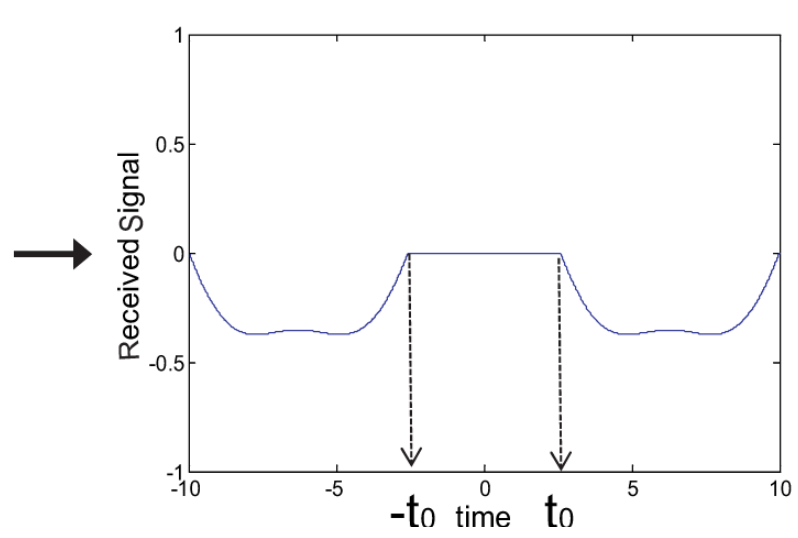

(b)

FIG. 8. (a) Example of received signal at $\mathrm{z}=0.1 \mathrm{~L}_{\mathrm{D}}$. $\mathrm{L}_{\mathrm{D}}$ is the dispersion length. (b) The output after a reverse diode function. 
CFC depending on the bit pattern and the pulse shape of the input signal is also seen in the work of Heismann [14]. Thus it could be said that the analytic study has its limitations, however we think that it can give us an inspiration as to how the CFC changes after each processing.

\section{CONCLUSION}

We reported an experimental demonstration and analytic derivation of the chromatic dispersion monitoring technique that we had proposed through numerical simulations in the earlier work. The proposed monitoring technique uses the CFC power extracted from the received NRZ signal. We showed that the experimental results of CFC power, monitoring range, and monitoring accuracy correspond with the simulation results quite well. Analytic derivation results also correspond with the experimental and simulation results. Therefore, we can say that the earlier work done by our numerical simulations are credible and the chromatic dispersion monitoring techniques based on CFC are feasible in the real optical transmission systems. We also demonstrated that the monitoring range and accuracy can be improved by using a simple clock-extraction method.

\section{ACKNOWLEDGMENT}

This research was supported by Kyungsung University Research Grants in 2012.

\section{REFERENCES}

1. T. Kato, Y. Koyano, and M. Nishimura, "Temperature dependence of chromatic dispersion in various types of optical fibers," in Proc. Optical Fiber Communication Conf. (Baltimore, USA, 2000), paper TuG7.

2. S. Kuwahara, A. Sano, K. Yonenaga, Y. Miyamoto, and H. Toba, "Simple zero dispersion detection technique using alternating chirp signal in automatic dispersion equalization system," Electron. Lett. 35, 593-594 (1999).

3. A. R. Chraplyvy, R. W. Tkach, L. L. Buhl, and R. C. Alferness, "Phase modulation to amplitude modulation conversion of $\mathrm{CW}$ laser light in optical fibres," Electron. Lett. 22, 409-411 (1986).

4. M. N. Petersen, Z. Pan, S. Lee, S. A. Havstad, and A. E. Willner, "Dispersion monitoring and compensation using a single inband subcarrier tone," in Proc. Optical Fiber Communication Conf. (Anaheim, USA, 2001), paper WH4.

5. G. Rossi, T. E. Dimmick, and D. J. Blumenthal, "Optical performance monitoring in reconfigurable WDM optical networks using subcarrier multiplexing," J. Lightwave Technol. 18, 1639-1648 (2000).

6. S.-M. Kim, "An algorithm for bit error rate monitoring and adaptive decision threshold optimization based on pseudo-error counting scheme," J. Opt. Soc. Korea 14, 22-27 (2010).

7. G. Ishikawa and H. Ooi, "Demonstration of automatic dispersion equalization in $40 \mathrm{Gbit} / \mathrm{s}$ OTDM transmission," in Proc. European Conf. on Optical Communication (Madrid, Spain, Sept. 1998), pp. 519-520.

8. Z. Pan, Q. Yu, Y. Xie, S. A. Havstad, A. E. Willner, D. S. Starodubov, and J. Feinberg, "Chromatic dispersion monitoring and automated compensation for NRZ and RZ data using clock regeneration and fading without adding signaling," in Proc. Optical Fiber Communication Conf. (Anaheim, USA, 2001), paper WH5.

9. K. J. Park, J. H. Lee, C. J. Youn, and Y. C. Chung, “A simultaneous monitoring technique for polarization-mode dispersion and group-velocity dispersion," in Proc. Optical Fiber Communication Conf. (Anaheim, USA, 2002), paper WE4.

10. A. Sano, T. Kataoka, M. Tomizawa, K. Hagimoto, K. Sato, K. Wakita, and K. Kato, "Automatic dispersion equalization by monitoring extracted-clock power level in a 40-Gbit/s, 200-km transmission line," in Proc. European Conf. on Optical Communication (Oslo, Norway, 1996), paper TuD.3.5.

11. S.-M. Kim, "PMD effect on the clock-based optimum dispersion compensation monitoring technique," J. Opt. Soc. Korea 13, 112-115 (2009).

12. S.-M. Kim and C.-H. Lee, "The efficient clock-extraction methods of NRZ signal for chromatic dispersion monitoring," IEEE Photon. Technol. Lett. 17, 1100-1102 (2005).

13. S.-M. Kim and J.-Y. Park, "Chromatic dispersion monitoring of CSRZ signal for optimum compensation using extracted clock-frequency component," ETRI Journal 30, 461-468 (2008).

14. F. Heismann, "Origin of clock-frequency components in NRZ-formatted optical signals," IEEE Photon. Technol. Lett. 15, $912-914$ (2003).

15. I. Kaminow and T. Li, Optical Fiber Telecommunications IV B (Academic Press, San Diego, USA, 2002), Chapter 16.

16. G. P. Agrawal, Nonlinear Fiber Optics, 4th ed. (Academic Press, New York, USA, 2007), Chapter 3.

17. E. W. Kamen, Introduction to Signals and Systems, 2nd ed. (Macmilian Publishing Company, New York, USA, 1990), Chapter 8 . 